\title{
BMJ Open Clinical value of chest pain presentation and prodromes on the assessment of cardiovascular disease: a cohort study
}

\author{
John Robson, ${ }^{1}$ Luis Ayerbe, ${ }^{1}$ Rohini Mathur, ${ }^{1}$ Juliet Addo, ${ }^{2}$ Andrew Wragg ${ }^{3}$
}

To cite: Robson J, Ayerbe L, Mathur $\mathrm{R}$, et al. Clinical value of chest pain presentation and prodromes on the assessment of cardiovascular disease: a cohort study. BMJ Open 2015;5:e007251. doi:10.1136/bmjopen-2014007251

- Prepublication history and additional material is available. To view please visit the journal (http://dx.doi.org/ 10.1136/bmjopen-2014007251).

Received 19 November 2014 Revised 19 January 2015 Accepted 1 February 2015

CrossMark

\footnotetext{
${ }^{1}$ Centre for Primary Care and Public Health, Queen Mary University of London, London, UK

${ }^{2}$ Department of Noncommunicable Disease Epidemiology, London School of Hygiene and Tropical Medicine, London, UK

${ }^{3}$ Department of Cardiology, Barts Health NHS Trust, London, UK
}

Correspondence to Dr John Robson; j.robson@qmul.ac.uk

\section{ABSTRACT}

Objectives: The recognition of coronary artery disease (CAD) among patients who report chest pain remains difficult in primary care. This study investigates the association between chest pain (specified, unspecified or musculoskeletal) and prodromes (dyspepsia, fatigue or dyspnoea), with firstever acute $\mathrm{CAD}$, and increased longer term cardiovascular risk.

Design: Cohort study.

Setting: Anonymised clinical data recorded electronically by general practitioners from 140 primary care surgeries in London (UK) between April 2008 and April 2013.

Participants: Data were extracted for all patients aged 30 years and over at the beginning of the study period, registered in the surgeries.

Main outcome measures: Clinical data included chest pain, dyspepsia, dyspnoea and fatigue, first-ever CAD and long-term cardiovascular risk (QRisk2).

Regression models were used to analyse the association between chest pain together with prodromes and CAD and QRisk $2 \geq 20 \%$.

Results: 354052 patients were included in the study. 4842 patients had first-ever CAD of which 270 reported chest pain in the year before the acute event. 257019 patients had QRisk2 estimations. Chest pain was associated with a higher risk of CAD. HRs: 21.12 (16.68 to 26.76), $p<0.001 ; 7.51$ (6.49 to 8.68), $p<0.001$; and 1.84 (1.14 to 3.00 ), $p<0.001$ for specified, unspecified and musculoskeletal chest pain. Dyspepsia, dyspnoea or fatigue was also associated with a higher risk of CAD. Chest pain of all subtypes, dyspepsia and dyspnoea were also associated with an increased 10-year cardiovascular risk of $20 \%$ or more. Conclusions: All patients with chest pain, including those with atypical symptoms, require careful assessment for acute and longer term cardiovascular risk. Prodromes may have independent diagnostic value in the estimation of cardiovascular disease risk.

\section{INTRODUCTION}

Coronary artery disease (CAD) is the leading cause of global mortality, accounting for $13 \%$ of deaths across the world. ${ }^{1}$ Chest pain is a common presenting symptom of CAD.
Strengths and limitations of this study

Chest pain of all clinical presentations (specified, unspecified and musculoskeletal) is associated with an increased risk of acute coronary artery disease (CAD) and increased longer term cardiovascular (CV) risk.

- Dyspepsia and dyspnoea in patients with chest pain are associated with acute CAD and longer term CV risk. Fatigue is also associated with increased risk of CAD.

- The data analysed in this study are derived from an almost complete population and not selected individuals or organisations; therefore, they provide the least biased sample frame and are likely to be representative of similar ethnically diverse populations.

- It was also possible to analyse several variables simultaneously, allowing the effect of multiple symptoms to be assessed.

- It is possible that this study underestimates the incidence of CAD as death outside the hospital may not always be recorded in the general practitioners' records. Patients who reported chest pain were more likely to have QRisk2 estimations; therefore, the association between the symptoms and the long-term CV risk may be overestimated.

However, the recognition of $\mathrm{CAD}$ among those who report chest pain remains difficult in primary care as chest pain only represents CAD in $8 \%^{2}$ to $11 \%^{3-5}$ of the patients. The guidelines of both the UK National Institute for Health and Care Excellence (NICE) and the American Heart Association acknowledge that clinical assessment may be sufficient to confirm or exclude the diagnosis of CAD. ${ }^{6} 7$ The NICE guidelines recommend the estimation of risk of CAD based on the typicality of the pain, age, gender and cardiovascular (CV) risk factors, and suggest the consideration of an alternative diagnosis if the estimated risk is below $10 \% .^{6}$ Many patients referred to secondary care are now offered investigations which have high costs and some involve exposure to ionising radiation. ${ }^{6}{ }^{8-10}$ This care pathway emphasises the relevance of the initial clinical assessment in 
primary care. Both unnecessary referral and failure to diagnose $\mathrm{CAD}$ are important issues from a resource and clinical outcome perspective. ${ }^{11}$

The initial clinical assessment in primary care should include the consideration of the typicality of chest pain. ${ }^{6}$ Clinicians appropriately refer central chest pain with shoulder radiation, or avoid referring typical musculoskeletal chest pain. However, there is a large grey area of unspecified chest pain that fits neither of these categories, particularly in older people at higher risk. The current recommendations for estimating the risk of CAD in patients with chest pain, acknowledging the typicality of symptoms, are based on a single secondary care study published in $1993 .{ }^{12}$ However, it is reported in the guidelines that this study may overestimate the risk of CAD in primary care. ${ }^{6} 12$ In addition, about half of the patients with $\mathrm{CAD}$ also report other prodromal symptoms to their general practitioner in the months before the acute event ${ }^{313-16}$ with dyspepsia, dyspnoea and fatigue among the most frequently reported. ${ }^{14}{ }^{16}$ There is little information on the diagnostic and prognostic value of such symptoms.

There are a number of validated tools, such as QRisk2, for the assessment of long-term CV risk in asymptomatic primary care patients. ${ }^{17}$ However, it is unclear whether these tools could also be used in patients with chest pain to estimate the risk of acute $\mathrm{CAD}$. We wished to undertake preliminary work to explore the association between type of chest pain, together with prodromal symptoms, and CV risk. This will inform subsequent research, using a larger data set, in which a tool for prediction of acute CAD in primary care patients with chest pain might be derived.

This paper investigates the association between chest pain (specified, unspecified and musculoskeletal) and prodromal symptoms (dyspepsia, dyspnoea and fatigue), with first-ever acute CAD. The association between chest pain and prodromal symptoms with longer term CV risk (QRisk2 scores) is also examined. ${ }^{17}$

\section{METHODS}

The study conformed to the STROBE (Strengthening the Reporting of Observational Studies in Epidemiology) study design recommendations. ${ }^{18}$

The study was based on patients living in three inner boroughs of east London (UK), registered with local general practitioners (GPs). This area had a population of 908096 in April 2013 and over half of the patients were of non-white ethnicity. Anonymised clinical and demographic data recorded in GPs' electronic health records between 1 April 2008 and 1 April 2013 were extracted using EMIS web software for all patients aged 30 years and over at the beginning of the study period. Patients from 140 of the 144 GP surgeries in the boroughs were used for the analysis (four surgeries used a different computer system and therefore were not included). Sociodemographic variables extracted included age, gender and self-reported ethnic group. The Townsend deprivation score, a measure of material deprivation derived from the UK Census at a small area level, that strongly correlates with standardised mortality ratios, was also recorded. ${ }^{19}$ Age was grouped into five categories of 35-44 years, 45-54, 55-64, 65-74, and 75 and older. Ethnicity was grouped into four categories: white, south Asian, black African/Caribbean and other. Individuals of mixed ethnicity were grouped with the relevant ethnic minority group. Clinical data included routinely recorded chest pain, prodromal symptoms and a report of a first-ever $\mathrm{CAD}$ in the electronic medical record. Chest pain was grouped into three categories of specified, unspecified and musculoskeletal. Table 1 shows the symptoms coded by the GPs that were used to define each of these categories in this study.

Prodromal symptoms included dyspepsia, dyspnoea and fatigue. The terms coded by the GPs that were used in this study to define $\mathrm{CAD}$ and each prodromal symptom are presented in online supplement 1. Additional clinical data used in the analyses included long-term CV risk, assessed with QRisk2 10-year CV risk scores. ${ }^{17}$ The QRisk2 score for prediction of $\mathrm{CV}$ disease within 10 years was stratified into lower $(0-9 \%)$, medium $(10-19 \%)$ and high risk $(\geq 20 \%) .{ }^{17}$

The associations between chest pain, dyspepsia, dyspnoea or fatigue and first-ever reported CAD in the year succeeding the onset of symptoms were investigated. $\mathrm{CAD}$ was reported in the year after the start of symptoms because it was considered both observations within that period of time could be assumed to be clinically related. When prodromes (dyspepsia, dyspnoea or fatigue) had been reported in the 12 months before chest pain, and they were independently associated with $\mathrm{CAD}$, they were combined with chest pain. The associations between the combination of chest pain and prodromes with first-ever $\mathrm{CAD}$ were also estimated.

Within the 5 years of the study period, the associations of chest pain, dyspepsia, dyspnoea or fatigue with high

Table 1 General practitioner notes used to define each category of chest pain

\begin{tabular}{ll}
$\begin{array}{l}\text { Chest pain } \\
\text { category }\end{array}$ & $\begin{array}{l}\text { Read codes from clinical notes } \\
\text { included in each variable }\end{array}$ \\
\hline Specified chest pain & $\begin{array}{l}\text { Ischaemic chest pain } \\
\text { Central chest pain } \\
\text { Precordial pain } \\
\text { Parasternal pain } \\
\text { Chest pain on exertion }\end{array}$ \\
& $\begin{array}{l}\text { Retrosternal pain } \\
\text { Anterior chest wall pain }\end{array}$ \\
Unspecified chest & $\begin{array}{l}\text { Chest wall pain } \\
\text { pain }\end{array}$ \\
& $\begin{array}{l}\text { Chest pain not otherwise specified } \\
\text { Atypical chest pain } \\
\text { Chest pain unspecified }\end{array}$ \\
& Rib pain \\
Musculoskeletal & Pleuritic pain \\
chest pain & Painful breathing-pleurodynia \\
\hline
\end{tabular}


$\mathrm{CV}$ risk (QRisk2 $\geq 20 \%)$ were investigated. When prodromes had been reported in the 12 months before chest pain, and they were independently associated with QRisk2 $>20 \%$, they were combined with chest pain. The associations between the combination of chest pain and prodromes with QRisk2>20\% were also estimated.

For patients with chest pain or prodromes recorded more than once, only the most recent episode of chest pain or the most recent prodrome, before the first-ever CAD or QRisk2 measurement, was included in the analysis. For those who had more than one QRisk2 estimation during the study period, only the first one was included in the analyses. Cox regression was used when $\mathrm{CAD}$ was the outcome. Cox regression describes how the risk of CAD changes over time in response to explanatory covariates (chest pain or prodromes). Results of Cox regression are presented in HRs, which are the expression of the risk of CAD occurring in patients with chest pain or prodromes as a ratio of the risk of CAD in patients without these symptoms. Models for the analysis of $\mathrm{CAD}$ were adjusted for age, gender, ethnicity and Townsend deprivation score. These variables were included in the models as they were considered potential confounders. Models for the analyses of QRisk2 were not adjusted as the QRisk2 algorithm already includes age, gender, ethnicity and Townsend score. SEs were adjusted for clustering by family practice.

\section{RESULTS}

In total, 354052 patients aged 30 years or older were included in the study. During the 5-year study period, $14222(4.0 \%)$ patients reported chest pain to their general practitioner, $70110(19.8 \%)$ patients had reported dyspnoea, dyspepsia or fatigue and 257019
(72.6\%) patients had a QRisk2 estimation. Table 2 shows the sociodemographic and clinical characteristics of patients with chest pain and prodromes.

Among the 4842 patients with first-ever CAD, 270 $(5.6 \%)$ reported chest pain to the general practitioner in the year before the acute event. Within the 12 months prior to the CAD event, 70 patients had a diagnosis of specified chest pain $(25.9 \%), 187$ had a diagnosis for unspecified chest pain $(69.2 \%)$ and 17 had a diagnosis for musculoskeletal pain $(6.3 \%)$. Some patients had more than one chest pain event coded in the preceding 12 months. For the purposes of calculating the risk of CAD after experiencing multiple chest pain events, the date of the chest pain closest to the CAD event was used. In the year preceding the CAD, 495 (10.2\%) patients had reported prodromes. Dyspepsia was reported by 232 (46.9\%) patients, dyspnoea by $235(47.5 \%)$ and fatigue by $54(10.9 \%)$ patients, with some patients having more than one prodrome recorded prior to CAD. Chest pain was independently (after adjusting for confounders) associated with a higher risk of CAD within the following year as shown in table 3 . This association was significant for all types of chest pain but strongest for patients reporting specified chest pain. Dyspepsia, dyspnoea or fatigue was also independently associated with a higher risk of $\mathrm{CAD}$ within the following year. The presence of chest pain of all subtypes in combination with dyspepsia, dyspnoea or fatigue was associated with an increased risk of CAD in the following year (table 3).

Among the 2027 patients with 10-year CV risk of $20 \%$ or more as defined by QRisk2 reported after chest pain or prodromes, prior to a QRisk2 value of $20 \%$ or greater being recorded on the patient record, $195(9.6 \%)$ had a diagnosis of specified chest pain, 1437 (70.9\%) had a diagnosis of unspecified chest pain and 492 had

Table 2 Characteristics of patients with chest pain and prodromes

\begin{tabular}{lllll}
\hline N=354 052 & Chest pain & Per cent & Prodromes & Per cent (95\% Cl) \\
\hline All patients aged 30+ on 1 April 2008 & 14222 & 100 & 70110 & 100 \\
Female & 7750 & 54.5 & 41402 & 59.1 \\
Age & & & 24447 & 34.9 \\
35-44 & 4480 & 31.5 & 19204 & 27.4 \\
45-54 & 4265 & 30.0 & 12392 & 17.7 \\
55-64 & 2876 & 20.2 & 7532 & 10.7 \\
65-74 & 1534 & 10.8 & 6535 & 9.3 \\
75+ & 1064 & 7.5 & 25277 & 36.1 \\
Ethnicity & & & 25709 & 36.7 \\
$\quad$ White & 5394 & 37.9 & 12987 & 18.5 \\
$\quad$ South Asian & 4863 & 34.2 & 3333 & 4.8 \\
Black & 2770 & 19.5 & 2804 & 4.0 \\
$\quad$ Other & 679 & 4.8 & 6.1 & $(-5.28$ to 9.58$)$ \\
Unknown & 516 & 3.6 & 495 & 0.71 (0.65 to 0.77$)$ \\
Townsend score (median and range) & 6.1 & $(-2.92$ to 9.68$)$ & 15007
\end{tabular}


Table 3 Risk of cardiovascular disease (CAD) within 1 year of chest pain, dyspepsia, dyspnoea or fatigue

\begin{tabular}{|c|c|c|c|c|c|c|}
\hline & $\mathbf{N}$ & $\begin{array}{l}\text { CAD with } \\
\text { in } 1 \text { year }\end{array}$ & $\begin{array}{l}\text { Median time } \\
\text { to CAD in } \\
\text { months (SD) }\end{array}$ & Adjusted HR & $(95 \% \mathrm{Cl})$ & p Value \\
\hline Any chest pain & 14222 & 270 & $3.3(3.1)$ & 2.32 & 2.11 to 2.56 & $<0.001$ \\
\hline Specified chest pain & 1136 & 70 & $2.6(2.8)$ & 21.12 & 16.68 to 26.76 & $<0.001$ \\
\hline Unspecified chest pain & 10226 & 187 & $3.3(3.0)$ & 7.51 & 6.49 to 8.68 & $<0.001$ \\
\hline Musculoskeletal chest pain & 3418 & 17 & $5.8(3.8)$ & 1.84 & 1.14 to 3.00 & 0.001 \\
\hline Dyspepsia & 45785 & 232 & $5.7(3.6)$ & 2.21 & 1.94 to 2.51 & $<0.001$ \\
\hline Dyspnoea & 14877 & 235 & $3.8(3.6)$ & 4.92 & 4.30 to 5.61 & $<0.001$ \\
\hline Fatigue & 19710 & 54 & $6.4(3.2)$ & 1.36 & 1.04 to 1.78 & 0.024 \\
\hline Any prodrome+specified chest pain & 134 & 5 & $3.3(1.5)$ & 12.84 & 5.34 to 30.87 & $<0.001$ \\
\hline Any prodrome+unspecified chest pain & 1098 & 37 & $3.8(3.0)$ & 11.17 & 8.08 to 15.45 & $<0.001$ \\
\hline Any prodrome+musculoskeletal pain & 335 & 8 & $10.8(23.1)$ & 2.52 & 1.26 to 5.04 & 0.009 \\
\hline
\end{tabular}

a diagnosis of musculoskeletal chest pain (24.3\%), with some patients having more than one chest pain event recorded. Of the 10337 patients who had $\mathrm{CV}$ risk of $20 \%$ or more after any prodrome, the most recent prodrome recorded was dyspepsia for 7022 (67.9\%), dyspnoea for 2865 (27.7\%) and fatigue for 1977 (19.1\%) cases, with some patients having more than one prodrome recorded. Chest pain of all subtypes was associated with a high CV risk of $20 \%$ or more. Dyspepsia and dyspnoea were also associated with a high CV risk of $20 \%$ or more. Finally, the presence of dyspepsia or dyspnoea in combination with chest pain of all subtypes was associated with a high long-term CV risk of $20 \%$ or more (table 4).

\section{DISCUSSION}

Our study suggests that all presentations of chest pain were associated with an increased risk of CAD, within the next year, and increased longer term CV risk. These associations were strongest for specified chest pain, but importantly were also present for other categories of chest pain. Dyspepsia and dyspnoea were also associated with CAD and longer term CV risk. Fatigue was associated with increased risk of CAD but not with longer term CV risk.

This study has strengths and weaknesses. The data are derived from an almost complete population and not selected individuals or organisations and are likely to be representative of similar ethnically diverse populations. The inclusion of the entire local population in a large data set provides the least biased sampling frame. In contrast with many previous articles, this study has a high number of cases and improved statistical power. $^{2} 1316$ It was also possible to analyse several variables simultaneously, allowing the effect of multiple symptoms to be assessed. CAD may have been overreported in our study, since we used an extensive list of terms for its definition. However, this broad definition of CAD probably minimised the number of CADs that were missed. The categorisation of chest pain used in this paper included pain location, which has been questioned as a predictor of CAD. ${ }^{20}$ Structured data entry templates and clinical facilitation in the east London practices studied enabled routine entry of high-quality data using agreed code sets for recording CV risk

Table 4 Odds of having QRisk2 $\geq 20 \%$ or more in patients with chest pain, dyspepsia, dyspnoea or fatigue

\begin{tabular}{|c|c|c|c|c|c|c|}
\hline & $\mathbf{N}$ & $\begin{array}{l}\text { QRisk2 } \\
\geq 20 \%\end{array}$ & $\begin{array}{l}\text { Median time to } \\
\text { QRisk2 } \geq 20 \% \text { in } \\
\text { months (SD) }\end{array}$ & Crude OR & $(95 \% \mathrm{CI})$ & p Value \\
\hline Any chest pain & 9134 & 2027 & $30.0(19.3)$ & 1.43 & 1.36 to 1.50 & $<0.001$ \\
\hline Specified chest pain & 688 & 195 & 31.9 (19.5) & 1.96 & 1.66 to 2.31 & $<0.00$ \\
\hline Unspecified chest pain & 6650 & 1437 & $31.2(19.7)$ & 1.37 & 1.29 to 1.45 & $<0.001$ \\
\hline Musculoskeletal chest pain & 2125 & 492 & 28.3 (18.3) & 1.49 & 1.35 to 1.65 & $<0.001$ \\
\hline Dyspepsia & 29492 & 7022 & $35.2(19.3)$ & 1.65 & 1.60 to 1.70 & $<0.001$ \\
\hline Dyspnoea & 8418 & 2865 & 30.2 (18.9) & 2.67 & 2.54 to 2.78 & $<0.001$ \\
\hline Fatigue & 12398 & 1977 & 30.4 (19.3) & 0.93 & 0.89 to 0.98 & 0.006 \\
\hline $\begin{array}{l}\text { Specified chest pain+dyspepsia or } \\
\text { dyspnoea }\end{array}$ & 99 & 20 & $34.4(16.7)$ & 2.53 & 1.48 to 4.34 & 0.001 \\
\hline $\begin{array}{l}\text { Unspecified chest pain+dyspepsia or } \\
\text { dyspnoea }\end{array}$ & 835 & 147 & $30.1(19.6)$ & 1.75 & 1.45 to 2.11 & $<0.001$ \\
\hline $\begin{array}{l}\text { Musculoskeletal chest pain+dyspepsia or } \\
\text { dyspnoea }\end{array}$ & 251 & 54 & 30.8 (15.3) & 2.30 & 1.66 to 3.18 & $<0.001$ \\
\hline
\end{tabular}


factors. Although CAD diagnoses were not adjudicated, the inclusion of this diagnosis is reviewed by local clinicians as part of their national Quality and Outcome Framework audit returns, which provide some validation of the diagnosis. It is possible that these underestimate total CAD as death outside hospital may not always be recorded in general practitioners' records.

Clinicians are likely to be aware that patients reporting specified chest pain have a greater risk of CAD than those with atypical symptoms. These patients will require assessment in secondary care in most cases. However, unspecified and musculoskeletal chest pain should not be disregarded as these symptoms are associated with the risk of CAD above $10 \%$. These results are consistent with the guidance for risk stratification ${ }^{12}$ included in the NICE guidelines. ${ }^{6}$ It has been reported that the association between atypical chest pain and CAD is strongest in older patients, south Asians and those affected by diabetes. ${ }^{21}$ Future studies may provide a more accurate estimation of the risk of CAD in primary care patients with chest pain in association with prodromal symptoms and other CV risk factors. It should also be noted that the proportion of patients with $\mathrm{CAD}$ who reported chest pain or prodromes to their GP in the year before the acute event was small. The natural history of CAD in the large proportion of patients who were not seen in primary care requires further clinical and epidemiological studies.

The factors that cause the association between chest pain and acute events are likely to explain its association with increased CV risk in the longer term. The typicality of symptoms appears to have less impact on the longer term CV risk. It is possible that some of the prodromal symptoms or episodes of chest pain observed within a year of CAD were not clinically related to it. Future studies may investigate whether the associations between chest pain, prodromes and CAD are similar when a shorter period of time (ie, three months) is observed. It should be noted that, in our sample, patients who reported chest pain were more likely to have QRisk2 estimations; therefore, the association between the symptoms and the long-term CV risk may be overestimated. The association between dyspepsia, dyspnoea or fatigue with CAD could also be partially explained by misdiagnosis in some patients, for example, angina being diagnosed as dyspepsia. ${ }^{22}$ Medication that prevents CV events, such as aspirin, may not have been prescribed to patients with dyspepsia, ${ }^{23}$ possibly increasing the rate of CAD among them.

A significant number of missed opportunities in the early management of CAD has been observed by other authors in primary care. ${ }^{16}$ Scores to estimate CV risk represent an attractive instrument to identify patients at high risk, both acutely and in the long term, and prompt more aggressive interventions. ${ }^{16}$ There are a number of reasons why the development of a risk score, using combinations of clinical data, ${ }^{24}$ for patients with chest pain in primary care may be appropriate. Patients with suspected $\mathrm{CAD}$ who are inadequately managed may have inappropriate and harmful investigations at high cost. Alternatively, those who are missed may have a preventable $\mathrm{CV}$ event. There are already validated and widely used scores predicting long-term $\mathrm{CV}$ risk in asymptomatic patients. ${ }^{17}$ There are also scores for risk assessment in patients with chest pain, which provide useful evidence for ruling out CAD. ${ }^{12}{ }^{25-30}$ However, only two of the available scores, routinely used in clinical practice, have been derived and validated in primary care. ${ }^{25} 26$ Electronic medical records represent clinical data sets of good quality, with larger samples than in most previous studies, ${ }^{11}$ and may provide a suitable basis to develop new and accurate scores for the diagnosis of acute and longer term CAD. The typicality of chest pain, together with the presence of dyspepsia and dyspnoea, might be considered in the further development of clinical decision rules based on risk of CAD for patients with chest pain but would require further validation.

Acknowledgements The study depended on the work and cooperation of general practitioners, practice staff and administrators in Newham, City and Hackney and Tower Hamlets' Clinical Commissioning Groups and the Clinical Effectiveness Group who support the east London EMIS web practice electronic health record database.

Contributors JR conceived the original idea. JR, LA, RM, JA and AW refined the questions and designed the study. RM conducted the analyses. JR, LA, $\mathrm{RM}, \mathrm{JA}$ and AW co-wrote the paper, gave final approval of the version to be published and agreed to be accountable for all aspects of the work in ensuring that questions related to the accuracy or integrity of any part of the work are appropriately investigated and resolved.

Funding The study was funded by a grant from the Worshipful Company of Curriers Millenium Bursary Fund.

Competing interests JR was an author of QRisk2, which predicts CVD risk in asymptomatic patients. The study was conducted independently of the funders.

Provenance and peer review Not commissioned; externally peer reviewed.

Data sharing statement No additional data are available.

Open Access This is an Open Access article distributed in accordance with the Creative Commons Attribution Non Commercial (CC BY-NC 4.0) license, which permits others to distribute, remix, adapt, build upon this work noncommercially, and license their derivative works on different terms, provided the original work is properly cited and the use is non-commercial. See: http:// creativecommons.org/licenses/by-nc/4.0/

\section{REFERENCES}

1. Lozano R, Naghavi M, Foreman K, et al. Global and regional mortality from 235 causes of death for 20 age groups in 1990 and 2010: a systematic analysis for the Global Burden of Disease Study 2010. Lancet 2012;380:2095-128.

2. Nilsson S, Scheike M, Engblom D, et al. Chest pain and ischaemic heart disease in primary care. Br J Gen Pract 2003;53:378-82.

3. Ruigomez A, Rodriguez LA, Wallander MA, et al. Chest pain in general practice: incidence, comorbidity and mortality. Fam Pract 2006;23:167-74

4. Klinkman MS, Stevens D, Gorenflo DW. Episodes of care for chest pain: a preliminary report from MIRNET. Michigan Research Network. J Fam Pract 1994;38:345-52.

5. Bösner S, Becker A, Haasenritter J, et al. Chest pain in primary care: epidemiology and pre-work-up probabilities. Eur J Gen Pract 2009;15:141-6.

6. Pathways: Acute Coronary Syndrome Overview. National Institute of Health and Care Excellence, 2013. http://pathways.nice.org.uk/ pathways/acute-coronary-syndromes 
7. Qaseem A, Fihn SD, Williams S, et al. Diagnosis of stable ischemic heart disease: summary of a clinical practice guideline from the American College of Physicians/American College of Cardiology Foundation/American Heart Association/American Association for Thoracic Surgery/Preventive Cardiovascular Nurses Association/ Society of Thoracic Surgeons. Ann Intern Med 2012;157:729-34.

8. O'Regan DP, Harden SP, Cook SA. Investigating stable chest pain of suspected cardiac origin. BMJ 2013;347:f3940

9. Radecki RP. CT coronary angiography: new risks for low-risk chest pain. Emerg Med J 2013;30:856-7.

10. Sarma A, Heilbrun ME, Conner KE, et al. Radiation and chest CT scan examinations: what do we know? Chest 2012;142:750-60.

11. Swap CJ, Nagurney JT. Value and limitations of chest pain history in the evaluation of patients with suspected acute coronary syndromes. JAMA 2005;294:2623-9.

12. Pryor DB, Shaw L, McCants CB, et al. Value of the history and physical in identifying patients at increased risk for coronary artery disease. Ann Intern Med 1993;118:81-90.

13. Madsen JK. Ischaemic heart disease and prodromes of sudden cardiac death. Is it possible to identify high risk groups for sudden cardiac death? Br Heart J 1985;54:27-32.

14. McSweeney JC, Cleves MA, Zhao W, et al. Cluster analysis of women's prodromal and acute myocardial infarction symptoms by race and other characteristics. J Cardiovasc Nurs 2010;25:311-22.

15. Then KL, Rankin JA, Fofonoff DA. Atypical presentation of acute myocardial infarction in 3 age groups. Heart Lung 2001;30:285-93.

16. Sequist TD, Marshall R, Lampert S, et al. Missed opportunities in the primary care management of early acute ischemic heart disease. Arch Intern Med 2006;166:2237-43.

17. Hippisley-Cox J, Coupland C, Vinogradova Y, et al. Predicting cardiovascular risk in England and Wales: prospective derivation and validation of QRISK2. BMJ 2008;336:1475-82.

18. Gallo V, Egger M, McCormack V, et al. STrengthening the Reporting of OBservational studies in Epidemiology-Molecular Epidemiology (STROBE-ME): an extension of the STROBE Statement. PLoS Med 2011;8:e1001117.

19. Jarman B, Townsend P, Carstairs V. Deprivation indices. BMJ 1991;303:523.
20. Bösner S, Bonisch $\mathrm{K}$, Haasenritter J, et al. Chest pain in primary care: is the localization of pain diagnostically helpful in the critical evaluation of patients? A cross sectional study. BMC Fam Pract 2013;14:154.

21. Sekhri N, Feder GS, Junghans C, et al. How effective are rapid access chest pain clinics? Prognosis of incident angina and non-cardiac chest pain in 8762 consecutive patients. Heart 2007;93:458-63.

22. Jaffery Z, Hudson MP, Khanal S, et al. The recognition of acute coronary ischemia in the outpatient setting. J Thromb Thrombolysis 2009;27:18-23.

23. Cleland JG, Alamgir F. Aspirin, myocardial infarction, and gastrointestinal bleeding. Lancet 1999;353:676.

24. Bruyninckx R, Aertgeerts B, Bruyninckx P, et al. Signs and symptoms in diagnosing acute myocardial infarction and acute coronary syndrome: a diagnostic meta-analysis. $\mathrm{Br} J$ Gen Pract 2008;58:105-11.

25. Bösner S, Haasenritter J, Becker A, et al. Ruling out coronary artery disease in primary care: development and validation of a simple prediction rule. CMAJ 2010;182:1295-300.

26. Gencer B, Vaucher P, Herzig L, et al. Ruling out coronary heart disease in primary care patients with chest pain: a clinical prediction score. BMC Med 2010;8:9.

27. Schillinger M, Sodeck G, Meron G, et al. Acute chest painidentification of patients at low risk for coronary events. The impact of symptoms, medical history and risk factors. Wien Klin Wochenschr 2004;116:83-9.

28. Sanchez M, Lopez B, Bragulat E, et al. Triage flowchart to rule out acute coronary syndrome. Am J Emerg Med 2007;25:865-72.

29. Sekhri N, Feder GS, Junghans C, et al. Incremental prognostic value of the exercise electrocardiogram in the initial assessment of patients with suspected angina: cohort study. BMJ 2008;337: a2240.

30. Martinez-Selles M, Bueno H, Sacristan A, et al. Chest pain in the emergency department: incidence, clinical characteristics and risk stratification. Rev Esp Cardiol 2008;61:953-9. 